\section{AN ELECTRONIC SOFTWARE AID FOR THE ASSESSMENT OF DEVELOPMENTAL MILESTONES IN CHILDREN}

M.O. Ogundele' ${ }^{1}$, H.F. Ayyash ${ }^{2}$

${ }^{1}$ Dept of Community Paediatrics, Alder Hey Childrens' Hospital NHS Foundation Trust, Liverpool, '2Dept of Paediatrics, Doncaster and Bassetlaw NHS Foundation Trust, Associated Teaching Hospitals, Doncaster, UK

Background: A child developmental screening covers the four key areas of gross motor, finemotor/ vision, hearing/ language and social skills. Medical students, GP's and paediatricians need a detailed understanding of both normal and abnormal patterns of developmental milestones. They also need a quick reference guide they can refer to in a busy clinical area.

Aim: We designed simple electronic software as reference guide for medical students and healthcare professionals when assessing the developmental milestones of children at different ages in a busy clinical area.

Design: The software is based on Microsoft windows application which is intuitively easy to use and user-friendly. It provides a database of information about normal pattern of developmental milestones with graphic illustration and warning red flags for possible developmental delay at different ages between birth and five years.

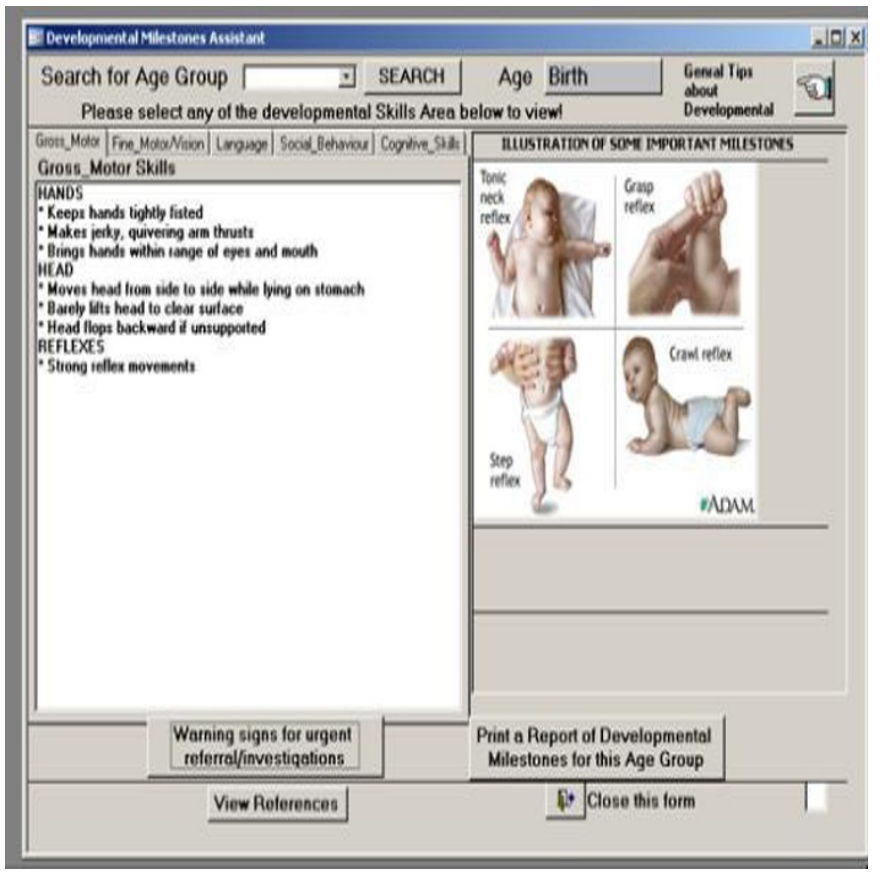

[Fig1]
Results: The computerised assessment tool acts as a quick reference for the approximate developmental age of children and requires no advanced computer skills. The software gives warning signals for developmental delay that should prompt immediate referral of children for developmental specialist review.

\section{Conclusion:}

The software programme can be utilized in the developmental assessment of preschool age children. It can identify children who might have developmental delay in the paediatric outpatient clinics or child development centres. Further study of its usefulness and effectiveness is recommended.

\section{0}

\section{MATERNAL AND TERM UMBILICAL CORD VITAMIN D LEVELS IN AN IRISH COHORT}

\author{
O. Uduma ${ }^{1}$, J. Brady ${ }^{2}$, R. McCarthy ${ }^{1}$, \\ M. McKenna ${ }^{2}$, B. Murray ${ }^{2}$, N. Murphy ${ }^{3}$, E. Molloy ${ }^{1}$ \\ ${ }^{1}$ Department of Neonatology, National Maternity \\ Hospital Holles Street, ${ }^{2}$ Metabolism Laboratory, \\ St Vincent's University Hospital, Dublin, ${ }^{3}$ Dept \\ Endocrinology, Children's University Hospital, \\ Dublin, Dublin, Ireland
}

Background and Object: Vitamin D plays an important role in skeletal and non skeletal health. We previously reported a high prevalence (about $80 \%$ ) of serum 25-hydroxy-vitamin D (25OHD) insufficiency in preterm VLBW infants. The object of this study was to assess 25OHD status in maternal and term umbilical cord samples.

Methods: Vitamin D levels were evaluated from maternal and umbilical cord samples from term normal pregnancies from January to March 2010 and evaluated by radioimmunoasssay.

Results: 40 samples included 19 maternal and 21 cord samples and 15 maternal and cord paired samples. The mean (+/-Standard deviation) cord blood $25 \mathrm{OHD}$ levels were significantly decreased compared with maternal levels $(29.9+/-8.3$ versus $47.9+/-22.0 \mathrm{nmol} / / \mathrm{p}<<0.005)$. Only one maternal and paired cord sample had levels $>70 \mathrm{nmol} / \mathrm{l}$.

Conclusions: We have demonstrated that $80 \%$ of preterm VLBW infants have insufficient 25OHD levels during early postnatal life; but $25 \%$ remain insufficient at follow-up assessment. However in addition a cohort of normal term mothers and infants 
have significantly decreased $250 \mathrm{HD}$ levels which may be related to the Irish climactic conditions.

1301

EARLY CHILDHOOD CARIES AND SOCIOECONOMIC ASPECTS IN CHILDREN UNDER 6 YEARS OLD IN THE NORTHEASTERN REGION OF BRAZIL

W.C. Reis ${ }^{1}$, V.S. Ribeiro ${ }^{2}$, M.C.N. Cruz ${ }^{3}$, E.B.F. Thomaz ${ }^{4}$, C.C.C. Ribeiro ${ }^{5}$

${ }^{1}$ Post Graduation in Collective Health, ${ }^{2}$ Medicine III - Post Graduation in Collective Health, University of Maranhao, ${ }^{3}$ Dentistry, University of Maranhão,

${ }^{4}$ Public Health - Post Graduation in Collective

Health, University of Maranhao, ${ }^{5}$ Dentistry, University of AarhusMaranhao, São Luís, Brazil

Aim: Verify association between socio-economic factors and early childhood caries in the Northeast region of Brazil.

\section{Methodology:}

It was carried out a cross-sectional study with 358 children from 6 to 71 months, from February 2008 to January 2009. The dependent variable was $\mathrm{dmft}$ index increased active white spot lesions; socioeconomic characteristics and care with the children were the independent variable. It had been used techniques of descriptive statistics and adjusted analysis through the Poisson's regression model for association with the socio-economic factors.

Results: The prevalence of the caries found was $46.9 \%$, being the age group from 37 to 71 months the ones more affect. Approximately $44.0 \%$ of the children belong to families with an income lower than a minimum wage. Limitations on the access to the health service $(18.4 \%)$, low coverage $(4.5 \%)$ and insufficient offer of preventive procedures $(23.7 \%)$ accentuate the prevalence of caries. It was observed the association among the children's age (RP 1.61), caries as reason for dental visit (RP 1.83) and the no use of fluorinated dental cream (RP 18.3) with manifested caries and activity of caries.

Conclusion: Socio-economic factors and disorganization of the public dental practice had influenced the sprouting and the non-homogeneous distribution of the caries, what must make this group a target of social policy and health.
1302

EPIDEMIOLOGY OF THE HYPOPLASTIC LEFT

HEART SYNDROME: IMPORTANCE OF AN ACCURATE DIAGNOSIS DURING PREGNANCY

V.P. Sheriff, M.S. Luna, D.B. Bravo, S. Caballero, M.L. Franco, B. Bernardo, E. Sanz, E. Zamora, C. Ramos, E. Maderuelo, S. Zeballos, M.I. Marsinyach, S. Villar, C.S. Gomez Orgaz, P. Chimenti, G. Zeballos, M. Arriaga

Pediatrics (neonatology), Hospital General Universitario Gregorio Marañon, Madrid, Spain

Aim: Infants with Hypoplastic Left Heart Syndrome (HLHS) may suffer inhibited growth. Some papers have shown it in congenital heart disease. Aim: to check if that is true in HLHS. We will also study other perinatal parameters that can be influenced by this pathology.

Methods: We have studied a total number of 173 HLHS of which $75(43,45 \%)$ died in our neonatal unit. We have compared data of maternal, pregnancy and delivery risks, weight, height and cephalic perimeter with normal babies born in the same period in our hospital,. We also compared the evolution between babies born at our hospital and those who were referred to us.

Results: No difference was obtained between normal and HLHS groups on: maternal age, number of previous gestations, abortion and live babies. There was significant difference in maternal pathology especially due to endocrine alterations (thyroid and diabetic). Also epilepsy was present. Both weight $(3216,05 \vee 2990,39 p<0,001)$ and cephalic perimeter $(34,26 \vee 33,41 p<0,001)$ was significantly different. Height had a $p<0,1$. At birth $\mathrm{pH}$, Apgar 1 minute were significantly diminished in the HLSH group.

HLSH born at our hospital had less morbidity and mortality.

Conclusions: We think it is important to diagnose pregnancy growth retardation and try to control it. As other authors have shown aortic arch morphometry during pregnancy should be controlled. Endocrine pathology or epilepsy in the mother may be a risk for HLSH. It is advisable that these babies should be born in reference hospitals. 